\title{
Democratic (S)fermions and Lepton Flavor Violation
}

\author{
K. Hamaguchi ${ }^{\mathrm{a}, \mathrm{b}, *}$, Mitsuru Kakizaki ${ }^{\mathrm{b}, \dagger}$ and Masahiro Yamaguchi ${ }^{\mathrm{b}, \ddagger}$ \\ a Deutsches Elektronen-Synchrotron DESY, D-22603, Hamburg, Germany \\ and \\ ${ }^{\mathrm{b}}$ Department of Physics, Tohoku University, Sendai 980-8578, Japan
}

(November 20, 2018)

\begin{abstract}
The democratic approach to account for fermion masses and mixing is known to be successful not only in the quark sector but also in the lepton sector. Here we extend this ansatz to supersymmetric standard models, in which the Kähler potential obeys underlying $S_{3}$ flavor symmetries. The requirement of neutrino bi-large mixing angles constrains the form of the Kähler potential for left-handed lepton multiplets. We find that right-handed sleptons can have non-degenerate masses and flavor mixing, while left-handed sleptons are argued to have universal and hence flavor-blind masses. This mass pattern is testable in future collider experiments when superparticle masses will be measured precisely. Lepton flavor violation arises in this scenario. In particular, $\mu \rightarrow e \gamma$ is expected to be observed in a planning future experiment if supersymmetry breaking scale is close to the weak scale.
\end{abstract}

\footnotetext{
*e-mail: koichi.hamaguchi@desy.de

$\dagger$ e-mail: kakizaki@tuhep.phys.tohoku.ac.jp

${ }^{\ddagger}$ e-mail: yama@tuhep.phys.tohoku.ac.jp
} 


\section{INTRODUCTION}

The flavor structure of quarks and leptons is one of the biggest puzzles of particle physics. The minimal Standard Model has 19 unexplained parameters, 13 of which are for masses and mixing of the fermions. A few more parameters (often associated with new particles) are needed to account for non-zero neutrino masses and their mixing. Some mechanism to explain the fermion mass structure is keenly desired.

The situation appears worse in supersymmetric (SUSY) extension of the Standard Model. The minimal SUSY Standard Model (MSSM) has additional 105 parameters, many of which are (SUSY breaking) masses and mixing of scalar partners of quarks and leptons, i.e. sfermions. Arbitrary mixing among the sfermions would cause too large flavor changing neutral current (FCNC) far above the experimental bounds: this problem is referred to as the SUSY flavor problem.

Thus the flavor problem in the SUSY Standard Model is two folds: 1) the puzzle in the fermion mass structure and 2) the puzzle in the sfermion mass structure. How these two are linked with each other is not clear. In fact attempts have been made to separate these issues and try to solve the latter independent of the former problem. This type of approach requires that the SUSY breaking occurring in a hidden sector is mediated in a special way to our Standard Model sector. The SUSY flavor problem can be solved in this way, though at the same time the resulting sfermion mass structure inherits no information on an underlying theory of flavor which is presumably a physics at very high energy scale.

Here we take an alternative approach to the flavor problem. Namely we shall consider the case where a mechanism which is responsible for the fermion mass structure also dictates the sfermion mass structure [1-7]. As was discussed earlier, such a strong connection between the two aspects of the flavor problem in supersymmetry is not mandatory. However this approach seems a natural one in the framework of generic supergravity where one does not impose specific features on the hidden sector and the messenger one. Moreover, if this idea indeed solves the SUSY flavor problem in nature, the structure of the sfermion masses reflects the mechanism how the fermion masses are generated, and thus one expects that future experiments will bring some hints towards our understanding of the theory of flavor.

Explicitly in this paper we consider a democratic type of fermion mass matrices (with diagonal breaking). The democratic ansatz [8-10] is very attractive because it can beautifully explain bi-large mixing in the neutrino sector while small mixing angles in the quark sector are kept. Moreover the underlying symmetry is a permutation group of three objects, $S_{3}$, which is one of the simplest groups and may be naturally realized in various settings. A particularly interesting possibility is that it can have a geometrical origin in brane-world scenario [11].

Here we assume that this democratic principle can also operate in the sfermion masses. More precisely the $S_{3}$ symmetry restricts possible forms of the Kähler potential, and thus

the scalar masses. Unlike the minimal supergravity, the resulting sfermion masses are not degenerate among different generations, but they are almost aligned with the fermion masses. A small violation of the democracy will induce rather suppressed flavor violation in the sfermion sector. Interestingly the effects to, e.g. $\mu \rightarrow e \gamma$ may be observed in near future experiments, as we will see later.

The paper is organized as follows. In the next section, we briefly review some basic 
points of the democratic ansatz for fermion masses. In Sec. 3, we extend this ansatz to the sfermion sector. We postulate that the Kähler potential is invariant under the $S_{3}$ transformations among the generations. This brings the characteristic mass spectrum for squarks and sleptons as well as their flavor mixing. The requirement that the neutrino sector has bi-large mixing angles yields very non-trivial constraints on the Kähler potential. In Sec. 4 , lepton flavor violation (LFV) is discussed. We show that it is plausible that $\mu \rightarrow e \gamma$ will be observed in near future experiments in our framework. In Sec. 5, we briefly discuss other flavor violation and $\mathrm{CP}$ violation in quark sector. The final section is devoted to conclusions and discussion.

\section{DEMOCRATIC FERMION MASSES}

In this section we briefly review the democratic ansatz for quarks and leptons [10]. We write the Yukawa interaction which generates the masses of quarks and charged leptons as follows:

$$
\begin{aligned}
W_{Y} & =Q_{L} \cdot Y_{q} Q_{R} H \\
& =Q_{L i}\left(Y_{q}\right)_{i j} Q_{R j} H,
\end{aligned}
$$

where $Q_{L i}$ and $Q_{R j}$ represent quarks or leptons of (left-handed) $\mathrm{SU}(2)_{L}$ doublets and (charge conjugated right-handed) $\mathrm{SU}(2)_{L}$ singlets, respectively, $H$ is a Higgs doublet and $\left(Y_{q}\right)_{i j}$ represent Yukawa coupling constants. Indices $i$ and $j$ stand for generations and run from 1 to 3 . Here we have used the notion of superpotential as well as chiral superfields for later convenience.

The basic idea of the democratic fermion is to postulate that the Yukawa matrix $Y_{q}$ approximately takes a democratic form

$$
Y_{q} \approx y_{0}^{q} J, \quad J \equiv \frac{1}{3}\left(\begin{array}{lll}
1 & 1 & 1 \\
1 & 1 & 1 \\
1 & 1 & 1
\end{array}\right),
$$

with some parameter $y_{0}^{q}$. It follows immediately that the Yukawa matrix is invariant under $S_{3}\left(Q_{L}\right) \times S_{3}\left(Q_{R}\right)$ transformation, where $S_{3}\left(Q_{L}\right)$ represents a permutation group among the three generations of $Q_{L i}$ 's, and $S_{3}\left(Q_{R}\right)$ of $Q_{R i}$ 's. We call the basis which gives (2) a democratic basis.

It is sometimes convenient to change the basis by a matrix

$$
A=\left(\begin{array}{ccc}
1 / \sqrt{2} & 1 / \sqrt{6} & 1 / \sqrt{3} \\
-1 / \sqrt{2} & 1 / \sqrt{6} & 1 / \sqrt{3} \\
0 & -2 / \sqrt{6} & 1 / \sqrt{3}
\end{array}\right) .
$$

We call this basis a canonical basis. In this basis the three-dimensional reducible representation $\underline{3}$ of $S_{3}$ is decomposed into a two-dimensional irreducible representation $\underline{2}$ and a one-dimensional (trivial) representation $\underline{1}$, namely $\underline{3}=\underline{2} \oplus \underline{1}$. The above mass matrix (2) becomes proportional to 


$$
T \equiv\left(\begin{array}{ccc}
0 & 0 & 0 \\
0 & 0 & 0 \\
0 & 0 & 1
\end{array}\right)
$$

where only the field corresponding to $\underline{1}$ has non-zero mass, which should be identified with the third generation in this democratic limit. ${ }^{1}$

To be more realistic, we need to include symmetry breaking terms. Let us first discuss the case of quarks. Following Ref. [9], we take in the democratic basis

$$
Y_{q}=y_{0}^{q}\left[J+\left(\begin{array}{ccc}
-\epsilon_{q} & 0 & 0 \\
0 & \epsilon_{q} & 0 \\
0 & 0 & \delta_{q}
\end{array}\right)\right] \text {, }
$$

where $q=u$ or $d$. Here we have assumed $\epsilon_{q} \ll \delta_{q} \ll 1$. An advantage of the breaking terms in the form given above becomes transparent if one moves to the canonical basis. There the first $2 \times 2$ entries of the matrix are of the Fritzsch type [12]:

$$
Y_{q}=y_{0}^{q}\left(\begin{array}{ccc}
0 & -\sqrt{1 / 3} \epsilon_{q} & -\sqrt{2 / 3} \epsilon_{q} \\
-\sqrt{1 / 3} \epsilon_{q} & (2 / 3) \delta_{q} & -(\sqrt{2} / 3) \delta_{q} \\
-\sqrt{2 / 3} \epsilon_{q} & -(\sqrt{2} / 3) \delta_{q} & 1+(1 / 3) \delta_{q}
\end{array}\right),
$$

from which one immediately obtains a phenomenologically favorable relation for the Cabibbo angle $\theta_{C}$ :

$$
\sin \theta_{C} \approx \sqrt{\frac{m_{d}}{m_{s}}}-\sqrt{\frac{m_{u}}{m_{c}}} \approx \sqrt{\frac{m_{d}}{m_{s}}}
$$

The mass matrix (5) is diagonalized by $U_{q}=A B_{q}$. The explicit form of $B_{q}$ is found in the literature $[9,10]$. Here it is sufficient to note that they are approximately

$$
B_{q}=\left(\begin{array}{ccc}
1 & O\left(\epsilon_{q} / \delta_{q}\right) & O\left(\epsilon_{q}\right) \\
O\left(\epsilon_{q} / \delta_{q}\right) & 1 & O\left(\delta_{q}\right) \\
O\left(\epsilon_{q}\right) & O\left(\delta_{q}\right) & 1
\end{array}\right)
$$

and the ratio of the mass eigenvalues is roughly given by

$$
m_{1}^{q}: m_{2}^{q}: m_{3}^{q} \approx \frac{\epsilon_{q}^{2}}{\delta_{q}}: \delta_{q}: 1
$$

A comparison with data determines the magnitudes of the breaking parameters. For instance, for the down-type quarks, we find

\footnotetext{
${ }^{1} S_{3}$ flavor symmetry was also considered in Ref. [7], where the three generations transform as $\underline{2} \oplus \underline{1}_{A}$ with $\underline{1}_{A}$ being a non-trivial one dimensional representation. The breaking pattern of the $S_{3}$ symmetry and hence the mass matrix in Ref. [7] is different from the democratic case we now consider.
} 


$$
\delta_{d} \sim 10^{-1}, \quad \epsilon_{d} \sim 10^{-2} .
$$

A similar expression can be obtained for the up-type quark sector, with much smaller breaking parameters:

$$
\delta_{u} \sim 10^{-2}, \quad \epsilon_{u} \sim 10^{-4} .
$$

The Cabibbo-Kobayashi-Maskawa (CKM) matrix can be obtained by

$$
U_{\mathrm{CKM}}=\left(A B_{u}\right)^{\dagger} A B_{d}=B_{u}^{\dagger} B_{d}
$$

Thus the matrix $A$ cancels out, and the remaining one is a matrix with small mixing angles. It has been argued that the CKM matrix obtained this way gives a phenomenologically successful pattern [9]. A detailed analysis of the quark masses and the CKM matrix in the democratic ansatz will be renewed elsewhere [13].

Let us now turn to the lepton sector. The Yukawa matrix of the charged leptons is assumed to be of the same form as those of the quarks [10]:

$$
Y_{l}=y_{0}^{l}\left[J+\left(\begin{array}{ccc}
-\epsilon_{l} & 0 & 0 \\
0 & \epsilon_{l} & 0 \\
0 & 0 & \delta_{l}
\end{array}\right)\right] .
$$

It possesses the flavor symmetry $S_{3}\left(L_{L}\right) \times S_{3}\left(E_{R}\right)$ where $S_{3}\left(L_{L}\right)$ and $S_{3}\left(E_{R}\right)$ stand for permutation symmetries among the three generations of $S U(2)_{L}$ doublet leptons and singlet leptons, respectively. The diagonalization can be performed in exactly the same manner as the case of the quarks. The mass ratio among the three generations implies

$$
\delta_{l} \sim 10^{-1}, \quad \epsilon_{l} \sim 10^{-2},
$$

like in the case of the down-type quarks.

The situation is quite different in the neutrino mass matrix, which is given by the bilinear of the doublet leptons. Thus it has two $S_{3}\left(L_{L}\right)$ invariants: $J$ and $I$, the latter of which denotes the $3 \times 3$ unit matrix. As was discussed by Ref. [10], the latter should be taken to obtain bi-large mixing angles which are strongly suggested by the atmospheric and the solar neutrino anomalies $[14-16]^{2}$. This choice may be justified in a brane-world scenario where each generation of the doublet leptons is localized on a different point in extra dimensions [11]. With diagonal breaking terms, we have the following mass matrix

$$
M_{\nu}=m_{\nu}\left[I+\left(\begin{array}{ccc}
-\epsilon_{\nu} & 0 & 0 \\
0 & \epsilon_{\nu} & 0 \\
0 & 0 & \delta_{\nu}
\end{array}\right)\right]
$$

We find the Maki-Nakagawa-Sakata (MNS) matrix is approximated to be $A^{T}$, and thus

$$
\sin ^{2} 2 \theta_{\text {solar }} \approx 1, \quad \sin ^{2} 2 \theta_{\text {atm }} \approx 8 / 9, \quad U_{e 3} \approx 0 .
$$

To summarize this section, the democratic ansatz with the diagonal breaking works well not only in the quark sector but also in the lepton sector.

\footnotetext{
${ }^{2}$ Very recently the first results from the KamLAND experiment have been announced, which exclude all solutions to the solar neutrino problem but the large-angle matter-enhanced one [17].
} 


\section{DEMOCRATIC SFERMIONS}

In this section we extend this democratic principle to the soft SUSY breaking terms. To begin with, let us recall the SUSY flavor problem in scenarios where supergravity interaction mediates the SUSY breaking: Arbitrary couplings in the Kähler potential between the visible sector and the hidden sector immediately cause disastrously large FCNC. There are several proposals to this problem. Some of the them attribute to separation between the two sectors by postulating special forms of the Kähler potential. On the other hand, in the minimal supergravity and its variants [18] a type of $\mathrm{U}(n)$ flavor symmetry in the Kähler metric, with $n$ being the number of generations, is implicitly assumed without any justification, while the Yukawa sector maximally breaks it. Here we will show that the Kähler potential controlled by the $S_{3}$ symmetry solves the SUSY flavor problem, and at the same time provides some interesting phenomenological consequences on the sfermion masses and mixing, which can be tested by future experiments.

The $S_{3}$ symmetry allows the following Kähler potential for the MSSM fields $Q_{i}$ :

$$
K=\left[Z_{I}^{Q} I+Z_{J}^{Q} J\right]_{i j} Q_{i}^{\dagger} Q_{j},
$$

where $Z_{I}^{Q}$ and $Z_{J}^{Q}$ are, in general, functions of (hidden sector) fields. In the following, we are interested in only their vacuum expectation values which can be expanded in terms of the Grassmann variables $\theta$ and $\bar{\theta}$ :

$$
Z_{I, J}^{Q}=z_{I, J}^{Q}+\left(\theta^{2} a_{I, J}^{Q}+\text { h.c. }\right)-\theta^{2} \bar{\theta}^{2} m_{Q I, J}^{2} .
$$

Notice that $a_{I, J}^{Q}$ and $m_{Q I, J}^{2}$ come from the SUSY breaking. The existence of the democratic part $Z_{J}^{Q}$ plays essential roles in our arguments. In fact, the non-universal Kähler metric coming from $z_{J}^{Q}$ leads to non-universal kinetic terms, and hence affects the fermion masses and their mixing. Furthermore the democratic part generates non-universal soft scalar masses, as we will explain in detail.

Using the matrix $A$ the Kähler metric for the fields $Q_{i}$ in eq. (17) is diagonalized as

$$
\left[z_{I}^{Q} I+z_{J}^{Q} T\right]_{i j}
$$

That is, we obtain the non-universal kinetic terms which are written explicitly

$$
K \supset\left(\begin{array}{ccc}
z_{I}^{Q} & 0 & 0 \\
0 & z_{I}^{Q} & 0 \\
0 & 0 & z_{I}^{Q}+z_{J}^{Q}
\end{array}\right)_{i j} Q_{i}^{\dagger} Q_{j}
$$

After rescaling the fields using the diagonal matrix

$$
C_{Q} \equiv \operatorname{diag}\left(1 / \sqrt{z_{I}^{Q}}, 1 / \sqrt{z_{I}^{Q}}, 1 / \sqrt{z_{I}^{Q}\left(1+r_{Q}\right)}\right), \quad r_{Q}=z_{J}^{Q} / z_{I}^{Q}
$$

we obtain canonically normalized kinetic terms. In the presence of $z_{J}^{Q}$, we call the field basis obtained this way the canonical basis.

Suppose that the Yukawa matrices have the same structure as the ones discussed in the previous section: 


$$
Y=y_{0}\left[J+\left(\begin{array}{ccc}
-\epsilon & 0 & 0 \\
0 & \epsilon & 0 \\
0 & 0 & \delta
\end{array}\right)\right]
$$

Diagonalization of the kinetic terms by $A$ with subsequent rescaling transforms the Yukawa matrix (22) into the one in the canonical basis, which is written

$$
Y=y_{0} C_{L}\left[T+A^{T}\left(\begin{array}{ccc}
-\epsilon & 0 & 0 \\
0 & \epsilon & 0 \\
0 & 0 & \delta
\end{array}\right) A\right] C_{R}
$$

where $C_{L, R}$ denote the rescaling matrices (21) for $Q_{L, R}$. Notice that in the canonical basis $Y(23)$ is already almost diagonalized.

The presence of the non-universal Kähler metric modifies the masses and mixing of the fermions. Let us consider the diagonalization of the Yukawa matrix $Y$ (23) given in the canonical basis:

$$
U_{L}^{T} Y U_{R}=\operatorname{diag}\left(y_{1}, y_{2}, y_{3}\right)
$$

It is easy to show that the eigenvalues are approximately

$$
y_{1}=Y_{0}\left(\frac{\Delta}{3}-\frac{\Xi}{6}\right), \quad y_{2}=Y_{0}\left(\frac{\Delta}{3}+\frac{\Xi}{6}\right), \quad y_{3}=Y_{0}\left(1+\frac{\delta}{3}\right)
$$

with

$$
\begin{aligned}
& Y_{0}=\frac{y_{0}}{\sqrt{z_{I}^{L} z_{I}^{R}\left(1+r_{L}\right)\left(1+r_{R}\right)}}, \quad \Xi=2\left(\Delta^{2}+3 E^{2}\right)^{1 / 2}, \\
& \Delta=\sqrt{\left(1+r_{L}\right)\left(1+r_{R}\right)} \delta, \quad E=\sqrt{\left(1+r_{L}\right)\left(1+r_{R}\right)} \epsilon .
\end{aligned}
$$

Also the unitary matrices $U_{L}$ and $U_{R}$ which diagonalize the matrix are found to be

$$
U_{L, R}=\left(\begin{array}{ccc}
\cos \theta & -\sin \theta & -\Lambda^{L, R} \sin 2 \theta \\
\sin \theta & \cos \theta & -\Lambda^{L, R} \cos 2 \theta \\
\Lambda^{L, R} \sin 3 \theta & \Lambda^{L, R} \cos 3 \theta & 1
\end{array}\right),
$$

where

$$
\tan 2 \theta=\frac{\sqrt{3} \epsilon}{\delta}, \quad \Lambda^{L, R}=\sqrt{1+r_{L, R}} \frac{\xi}{3 \sqrt{2}}, \quad \xi=2\left(\delta^{2}+3 \epsilon^{2}\right)^{1 / 2}
$$

As far as the normalization factor $1+r_{L, R}$ is of order unity, the masses and mixing angles of the quarks and charged leptons are not drastically modified. On the other hand, this factor is very important for the neutrino mixing, as we will discuss later.

Let us now discuss the soft SUSY breaking terms. After the $A$ rotation, soft scalar mass terms for sfermions $\tilde{q}_{i}$ are written

$$
\begin{aligned}
& -\mathcal{L} \supset\left(m_{Q}^{2}\right)_{i j} \tilde{q}_{i}^{\dagger} \tilde{q}_{j}, \\
& m_{Q}^{2}=\left(m_{Q I}^{2} I+m_{Q J}^{2} T\right)+\left(a_{I}^{Q} I+a_{J}^{Q} T\right)\left(z_{I}^{Q} I+z_{J}^{Q} T\right)^{-1}\left(a_{I}^{Q} I+a_{J}^{Q} T\right) .
\end{aligned}
$$


Since the second term of $m_{Q}^{2}$ is rewritten in terms of the linear combination of $I$ and $T$, hereafter we absorb this term into $m_{Q I, J}^{2}$. To go to the canonical basis, we need to multiply $C_{Q}$ from both sides, so that they become

$$
\begin{aligned}
m_{Q}^{2} & =C_{Q}\left(m_{Q I}^{2} I+m_{Q J}^{2} T\right) C_{Q} \\
& =m_{Q 0}^{2}\left[\left(\begin{array}{lll}
1 & 0 & 0 \\
0 & 1 & 0 \\
0 & 0 & 1
\end{array}\right)+\rho_{Q}\left(\begin{array}{lll}
0 & 0 & 0 \\
0 & 0 & 0 \\
0 & 0 & 1
\end{array}\right)\right], \quad m_{Q 0}^{2}=\frac{m_{Q I}^{2}}{z_{I}^{Q}}, \quad \rho_{Q}=\frac{z_{I}^{Q} m_{Q J}^{2}-z_{J}^{Q} m_{Q I}^{2}}{\left(z_{I}^{Q}+z_{J}^{Q}\right) m_{Q I}^{2}}
\end{aligned}
$$

We find that the quantity $\rho_{Q}$ becomes non-zero if the ratio of the democratic part to the unit part in the $D$-components of $Z, m_{Q J}^{2} / m_{Q I}^{2}$, is different from the same ratio in the lowest components, $r_{Q}=z_{J}^{Q} / z_{I}^{Q}$. We expect that this is generically the case and thus $\rho_{Q}$ is of order unity. The existence of the second term of the mass-squared matrix leads to very interesting consequences. As one can readily see from eq. (30), the third generation sfermion has a different mass than the degenerate first two generation masses, that is

$$
m_{1 s t}^{2}=m_{2 n d}^{2} \neq m_{3 r d}^{2} .
$$

Furthermore, in the fermion-mass basis where the fermion masses are diagonal, there arise non-zero off diagonal elements in the sfermion mass-squared matrix, leading to flavor mixing mediated by the sfermions. In fact, in this basis, the mass-squared matrix becomes of the following form:

$$
\begin{aligned}
m_{Q}^{2} & =m_{Q 0}^{2}\left[\left(\begin{array}{lll}
1 & 0 & 0 \\
0 & 1 & 0 \\
0 & 0 & 1
\end{array}\right)+\rho_{Q}\left(\begin{array}{ccc}
\Lambda^{2} \sin ^{2} 3 \theta & \Lambda^{2} \sin 3 \theta \cos 3 \theta & \Lambda \sin 3 \theta \\
\Lambda^{2} \sin 3 \theta \cos 3 \theta & \Lambda^{2} \cos ^{2} 3 \theta & \Lambda \cos 3 \theta \\
\Lambda \sin 3 \theta & \Lambda \cos 3 \theta & 1
\end{array}\right)\right], \\
& \sim m_{Q 0}^{2}\left(\begin{array}{ccc}
1 & O(\rho \Delta E) & O(\rho E) \\
O(\rho \Delta E) & 1 & O(\rho \Delta) \\
O(\rho E) & O(\rho \Delta) & 1+\rho
\end{array}\right), \quad \rho \sim O(1),
\end{aligned}
$$

where the coefficients in each entries can be computed by using eq. (27) and $\Lambda$ here corresponds to $\Lambda_{L}$ or $\Lambda_{R}$ in eq. (28)

Let us next turn to scalar trilinear couplings, i.e. A-terms. Following the same procedure, we find that the $A$-terms in the canonical basis are given as

$$
-\mathcal{L} \supset \sum_{Q} \frac{\partial W}{\partial \tilde{q}_{i}}\left[\left(z_{I}^{Q} I+z_{J}^{Q} T\right)^{-1}\left(a_{I}^{Q} I+a_{J}^{Q} T\right)\right]_{i j} \tilde{q}_{j} .
$$

As in the previous case, disagreement between $z_{J}^{Q} / z_{I}^{Q}$ and $a_{J}^{Q} / a_{I}^{Q}$ induces small off-diagonal elements in the fermion-mass basis, resulting in again the flavor mixing. An inspection shows that the above equation is written

$$
\sum_{Q} \frac{\partial W}{\partial \tilde{q}_{i}}\left[a_{0}^{Q} I+a_{1}^{Q} T\right]_{i j} \tilde{q}_{j}
$$

where 


$$
a_{0}^{Q}=\frac{a_{I}^{Q}}{z_{I}^{Q}}, \quad a_{1}^{Q}=\frac{a_{J}^{Q} z_{I}^{Q}-a_{I}^{Q} z_{J}^{Q}}{\left(z_{I}^{Q}+z_{J}^{Q}\right) z_{I}^{Q}} .
$$

With the superpotential of the form $W=Q_{L} \cdot Y Q_{R} H$, eq. (34) reduces to

$$
\left(a_{0}^{L}+a_{0}^{R}+a_{0}^{H}\right) \tilde{q}_{L} \cdot Y \tilde{q}_{R} H+a_{1}^{L} \tilde{q}_{L} \cdot T Y \tilde{q}_{R} H+a_{1}^{R} \tilde{q}_{L} \cdot Y T \tilde{q}_{R} H .
$$

One can move to the fermion mass basis by multiplying $U_{L}^{T}$ and $U_{R}$ from left and right, respectively. The matrix for the trilinear couplings becomes of the following form

$$
\begin{aligned}
A_{q}= & \left(a_{0}^{L}+a_{0}^{R}+a_{0}^{H}\right)\left(\begin{array}{ccc}
y_{1} & 0 & 0 \\
0 & y_{2} & 0 \\
0 & 0 & y_{3}
\end{array}\right)+a_{1}^{L}\left(U_{L}^{T} T U_{L}^{*}\right)\left(\begin{array}{ccc}
y_{1} & 0 & 0 \\
0 & y_{2} & 0 \\
0 & 0 & y_{3}
\end{array}\right) \\
& +a_{1}^{R}\left(\begin{array}{ccc}
y_{1} & 0 & 0 \\
0 & y_{2} & 0 \\
0 & 0 & y_{3}
\end{array}\right)\left(U_{R}^{\dagger} T U_{R}\right),
\end{aligned}
$$

and

$$
\begin{aligned}
U_{L, R}^{\dagger} T U_{L, R} & =\left(\begin{array}{ccc}
\left(\Lambda^{L, R}\right)^{2} \sin ^{2} 3 \theta & \left(\Lambda^{L, R}\right)^{2} \sin 3 \theta \cos 3 \theta & \left(\Lambda^{L, R}\right)^{2} \sin 3 \theta \\
\left(\Lambda^{L, R}\right)^{2} \sin 3 \theta \cos 3 \theta & \left(\Lambda^{L, R}\right)^{2} \cos ^{2} 3 \theta & \Lambda^{L, R} \cos 3 \theta \\
\Lambda^{L, R} \sin 3 \theta & \Lambda^{L, R} \cos 3 \theta & 1
\end{array}\right) \\
& \sim\left(\begin{array}{ccc}
E^{2} & \Delta E & E \\
\Delta E & \Delta^{2} & \Delta \\
E & \Delta & 1
\end{array}\right) .
\end{aligned}
$$

We find that the flavor mixing terms will not become symmetric matrices. This will play an important role when discussing lepton flavor violation.

Following Ref. [19], we now introduce some notation on the sfermion mass terms in the fermion mass basis

$$
\left(m_{L L}^{2}\right)_{i j} \tilde{q}_{L i}^{*} \tilde{q}_{L j}+\left(m_{R R}^{2}\right)_{i j} \tilde{q}_{R i}^{*} \tilde{q}_{R j}+\left(m_{L R}^{2}\right)_{i j} \tilde{q}_{L i}^{*} \tilde{q}_{R j}+\left(m_{R L}^{2}\right)_{i j} \tilde{q}_{R i}^{*} \tilde{q}_{L j}, \quad m_{L R}^{2 \dagger}=m_{R L}^{2}
$$

where each is given as

$$
\begin{aligned}
& \left(m_{L L}^{2}\right)_{i j}=\left(m_{Q_{L}}^{2}\right)_{i j}, \quad\left(m_{R R}^{2}\right)_{i j}=\left(m_{Q_{R}}^{2 T}\right)_{i j}, \\
& \left(m_{L R}^{2}\right)_{i j}=\left(A_{y}^{*}\right)_{i j}\langle H\rangle, \quad\left(m_{R L}^{2}\right)_{i j}=\left(A_{y}^{T}\right)_{i j}\langle H\rangle .
\end{aligned}
$$

Here we have written only SUSY breaking masses explicitly, and suppressed SUSY contributions. For later use, it is convenient to define

$$
\begin{array}{ll}
\left(\delta_{i j}\right)_{L L} \equiv \frac{\left(m_{L L}^{2}\right)_{i j}}{m^{2}}, & \left(\delta_{i j}\right)_{R R} \equiv \frac{\left(m_{R R}^{2}\right)_{i j}}{m^{2}}, \\
\left(\delta_{i j}\right)_{L R} \equiv \frac{\left(m_{L R}^{2}\right)_{i j}}{m^{2}}, & \left(\delta_{i j}\right)_{R L} \equiv \frac{\left(m_{R L}^{2}\right)_{i j}}{m^{2}},
\end{array}
$$

where $m$ represents a typical mass of the corresponding sfermion.

In this paper, we assume that the Kähler potential is invariant under the $S_{3}$ symmetry and do not include possible $S_{3}$ breaking contributions. This assumption is, however, not stable against radiative corrections. In particular, renormalization group effects involving the Yukawa couplings will violate the symmetry in the Kähler potential, which generate different patterns of violations of mass degeneracy and flavor mixing. We will return to this point in the subsequent sections. 


\section{A. (S)lepton Sector}

In this subsection, we would like to focus on the lepton sector and discuss the slepton masses in detail.

Following the general argument given above, we can perform the diagonalization of the Yukawa matrix for the charged leptons. The results are

$$
y_{e}=Y_{0}^{l}\left(\frac{\Delta_{l}}{3}-\frac{\Xi_{l}}{6}\right), \quad y_{\mu}=Y_{0}^{l}\left(\frac{\Delta_{l}}{3}+\frac{\Xi_{l}}{6}\right), \quad y_{\tau}=Y_{0}^{l}\left(1+\frac{\delta_{l}}{3}\right),
$$

and

$$
U_{L, R}=\left(\begin{array}{ccc}
\cos \theta_{l} & -\sin \theta_{l} & -\Lambda^{L, R} \sin 2 \theta_{l} \\
\sin \theta_{l} & \cos \theta_{l} & -\Lambda^{L, R} \cos 2 \theta_{l} \\
\Lambda^{L, R} \sin 3 \theta_{l} & \Lambda^{L, R} \cos 3 \theta_{l} & 1
\end{array}\right)
$$

Next we consider the neutrino mass matrix. We assume that the neutrino mass matrix is the same as the one discussed in Section 2 (eq. (15)). It becomes, in the canonical basis,

$$
M_{\nu}=m_{\nu} C_{L}\left[\left(\begin{array}{ccc}
1 & 0 & 0 \\
0 & 1 & 0 \\
0 & 0 & 1
\end{array}\right)+A^{T}\left(\begin{array}{ccc}
0 & 0 & 0 \\
0 & \epsilon_{\nu} & 0 \\
0 & 0 & \delta_{\nu}
\end{array}\right) A\right] C_{L}
$$

Without $C_{L}$, the first term, i.e. the unit matrix, of eq. (44) is irrelevant to the diagonalization, and thus the mass matrix itself is diagonalized by the matrix $A$. It is this fact which realizes the bi-large mixing angles. However, when $r_{L}$ is non-zero and $C_{L}$ is not proportional to the unit matrix, this is no longer the case. This is because the first term in eq (44) is proportional to $\operatorname{diag}\left(1,1,1+r_{L}\right)$ and the diagonalizing matrix is different from $A$. In fact, it is an easy task to see that the whole mass matrix in this case is diagonalized by a matrix close to the unit matrix (with small angles). Combining the fact that the diagonalizing matrix for the charged lepton sector contains only small mixing angles, we find that mixing angles of the neutrinos would be all small when the $r_{L}$ is sizably different from zero. On the other hand, in the limit of $r_{L} \ll \epsilon_{\nu}, \delta_{\nu}$, the first term in (44) can be regarded as a unit matrix. Then the unitary matrix arises only from the second term in (44) and equals to $A$, from which we can reproduce bi-large mixing angles in the neutrino sector. We conclude that the absence of the democratic part in the Kähler metric $z_{J}^{L}$ of the doublet leptons is required by the neutrino phenomenology.

This observation strongly implies that the whole democratic part of the Kähler potential for the doublet leptons, namely $Z_{J}^{L}=z_{J}^{L}+\left(\theta^{2} a_{J}^{L}+\right.$ h.c. $)-\theta^{2} \bar{\theta}^{2} m_{L J}^{2}$, should be absent. Though the absence of only the lowest component, $z_{J}^{L}$, is sufficient for the bi-large mixing to be realized, we argue that it is unnatural and thus unlikely. Assuming the absence of the whole democratic part, namely $z_{J}^{L}=a_{J}^{L}=m_{L J}^{2}=0$, we immediately conclude that all left-handed slepton masses are degenerate:

$$
m_{\tilde{e}_{L}}^{2}=m_{\tilde{\mu}_{L}}^{2}=m_{\tilde{\tau}_{L}}^{2},
$$

at the energy scale where these soft scalar masses are given, presumably at a scale close to the Planck scale. At the same time there will be no flavor mixing in the left-handed slepton 
mass-squared matrix. On the other hand, the right-handed lepton sector generically has the democratic part in its Kähler potential, and thus the right-handed stau has a different mass from those in the first two generations:

$$
m_{\tilde{e}_{R}}^{2}=m_{\tilde{\mu}_{R}}^{2} \neq m_{\tilde{\tau}_{R}}^{2}
$$

Furthermore its presence also leads flavor mixing of the right-handed slepton mass-squared matrix of the slepton sector. As for the $A$-terms, the absence of the democratic part for the left-handed leptons implies $a_{1}^{L}=0$, and thus the matrix for the $A$-terms (37) reduces to

$$
A_{l}=\left(a_{0}^{L}+a_{0}^{R}+a_{0}^{H}\right)\left(\begin{array}{ccc}
y_{e} & 0 & 0 \\
0 & y_{\mu} & 0 \\
0 & 0 & y_{\tau}
\end{array}\right)+a_{1}^{R} Y_{0}^{l}\left(\begin{array}{ccc}
O\left(E^{4} / \Delta\right) & O\left(E^{3}\right) & O\left(E^{3} / \Delta\right) \\
O\left(\Delta^{2} E\right) & O\left(\Delta^{3}\right) & O\left(\Delta^{2}\right) \\
O(E) & O(\Delta) & 1
\end{array}\right)
$$

It is straightforward to compute numerical values of the matrix in the second term, once $\Delta_{l}$ and $E_{l}$ are fixed by the charged lepton masses ${ }^{3}$

$$
\Delta_{l} \approx \frac{3 m_{\mu}}{2 m_{\tau}-m_{\mu} / \sqrt{1+r_{R}}}, \quad E_{l} \approx \sqrt{\frac{4 m_{e}}{3 m_{\mu}}} \Delta_{l} .
$$

Notice that they are insensitive to the choice of $r_{R}$ as far as $\sqrt{1+r_{R}}$ is of order unity.

The mass spectrum in eqs. (45) and (46) is characteristic of the democratic sfermion ansatz. In fact the degeneracy of the left-handed slepton masses is strongly suggested by realizing the bi-large neutrino mixing angles. Such a constraint does not come to the righthanded sector, and thus the right-handed stau can have a different mass which is consistent with the $S_{3}$ symmetry. This situation should be compared with the renormalization group (RG) effect due to right-handed neutrino Yukawa couplings, which leads the non-degeneracy in the left-handed slepton masses [20]. The non-degeneracy in the right-handed sector can also be induced in SU(5) grand unified theories (GUTs) by the RG effect above the GUT scale [21]. One possible way to distinguish our case with the GUT contribution is that in our case the right-handed stau can be either light or heavy, while the RG flow always makes the right-handed stau lighter than the other generations.

\section{LEPTON FLAVOR VIOLATION}

We are now at a position to discuss lepton flavor violation. FCNC processes in the quark sector will be briefly discussed in the subsequent section. In our setup, we will show that the predicted rate of $\mu \rightarrow e \gamma$ is within reach of planning experiments as far as the sparticle masses are of order $100 \mathrm{GeV}$. We also find that the rate of $\tau \rightarrow \mu \gamma$ is smaller than the present experimental bound by at least two orders of magnitudes.

In SUSY models, superparticle exchange loop diagrams induce the dipole moment operators

\footnotetext{
${ }^{3}$ We neglect $r_{L}$ since it is extremely small.
} 


$$
\mathcal{L}_{\mathrm{eff}}=e \frac{m_{l_{j}}}{2} \bar{l}_{i} \sigma_{\mu \nu} F^{\mu \nu}\left(L_{i j} P_{L}+R_{i j} P_{R}\right) l_{j}
$$

where $m_{l_{j}}$ denotes a charged lepton mass of generation $j, P_{L, R}=\left(1 \mp \gamma^{5}\right) / 2$ indicate the chiral projection operators, and $L_{i j}$ and $R_{i j}$ are coefficients dictated by the parameters in the SUSY models. Flavor violating processes take place if off-diagonal components in scalar mass matrices exist. For lepton flavor violation, the present experimental bound [22] $\operatorname{Br}(\mu \rightarrow e \gamma)<1.2 \times 10^{-11}$ constrains the off-diagonal elements of the slepton masses. Here we give an estimate by using an approximate formula similar to Ref. [19], where we only consider the diagrams given in Fig. 1 with Bino and Wino loops. More details will be found in Appendix. The constraints obtained in this approximation are

$$
\begin{aligned}
\left(\delta_{12}^{l}\right)_{L L} & \lesssim(\text { a few }) \times 10^{-3}\left(\frac{m_{\tilde{l}}}{100 \mathrm{GeV}}\right)^{2}, \\
\left(\delta_{12}^{l}\right)_{R R} & \lesssim(\text { a few }) \times 10^{-3}\left(\frac{m_{\tilde{l}}}{100 \mathrm{GeV}}\right)^{2}, \\
\left(\delta_{12}^{l}\right)_{L R}, \quad\left(\delta_{12}^{l}\right)_{R L} & \lesssim(\text { a few }) \times 10^{-6}\left(\frac{m_{\tilde{l}}}{100 \mathrm{GeV}}\right)^{2},
\end{aligned}
$$

where $m_{\tilde{l}}$ represents a characteristic mass scale of the sleptons, and the constraints get weaker as $m_{\tilde{l}}$ increases. We note that exact numbers are sensitive to the ratios of the gaugino masses and the slepton masses, and the values given above should be taken as typical ones.

These constraints should be compared with the expected values of the off-diagonal elements in the framework of the democratic sfermions. For $m_{\tilde{l}} \sim 100 \mathrm{GeV}$ and the scalar trilinear parameter $a_{1}^{R} \sim m_{\tilde{l}}$, we find

$$
\begin{aligned}
& \left(\delta_{12}^{l}\right)_{L L} \sim 0, \quad\left(\delta_{12}^{l}\right)_{R R} \sim \rho_{R} \Delta_{l} E_{l} \sim 10^{-3}, \\
& \left(\delta_{12}^{l}\right)_{L R} \sim \frac{a_{1}^{R} \Delta_{l} E_{l} m_{e}}{m_{\tilde{l}}^{2}} \sim 10^{-8}, \quad\left(\delta_{12}^{l}\right)_{R L} \sim \frac{a_{1}^{R} \Delta_{l} E_{l} m_{\mu}}{m_{\tilde{l}}^{2}} \sim 10^{-6} .
\end{aligned}
$$

The comparison shows that the flavor mixing expected in our framework is already comparable with the experimental constraints if the SUSY breaking scale is around the weak scale. Put another way, we naturally expect that the $\mu \rightarrow e \gamma$ process will be seen in a near future experiment if the sparticles in the loops have masses of order $100 \mathrm{GeV}$.

The same comparison also indicates that the off-diagonal mixing components $\left(\delta_{12}\right)_{R R}$ and $\left(\delta_{12}\right)_{R L}$ give comparable and dominant contributions to $\mu \rightarrow e \gamma$. Both contributions generate non-zero $L_{12}$ in eq. (49), with vanishing $R_{12}$. It is interesting to compare this with other scenarios of lepton flavor violation. For instance, $\left(m_{L L}^{2}\right)_{12}$ dominates when the RG effect due to the right-handed neutrino Yukawa interactions gives considerable flavor violation [23,24]. In this case, only $R_{12}$ becomes non-zero. On the other hand, $\left(m_{R R}^{2}\right)_{12}$ will dominate in the minimal SU(5) GUT if the RG contributions above the GUT scale are important [21], which gives non-zero $L_{12}$ term, as in our scenario. It is interesting to note that the case with non-zero $L_{12}$ may be distinguishable from the other case with non-zero $R_{12}$ if polarized muon beam is available, by measuring the angular distribution of the decaying particles [25].

Next we would like to present some numerical results. We calculate the rate of the $\mu \rightarrow e \gamma$ by using the formulae presented in Appendix. For simplicity, we take a universal gaugino mass $M_{1 / 2}$ : 


$$
M_{\widetilde{B}}\left(M_{X}\right)=M_{\widetilde{W}}\left(M_{X}\right)=M_{\widetilde{G}}\left(M_{X}\right)=M_{1 / 2},
$$

where $M_{\widetilde{B}}, M_{\widetilde{W}}$ and $M_{\widetilde{G}}$ represent bino, wino and gluino masses respectively, and assume the equality of the right-handed slepton masses in the first two generations and the lefthanded slepton masses $m_{R 0}^{2}\left(M_{X}\right)=m_{L 0}^{2}\left(M_{X}\right)=m_{0}^{2}$ at the boundary $M_{X}=2 \times 10^{16} \mathrm{GeV}$. The masses at low energy are obtained with the help of renormalization group equations. Relevant quantities in our analysis are

$$
\begin{aligned}
M_{\widetilde{B}}(\mu) & =0.43 M_{1 / 2}, \quad M_{\widetilde{W}}(\mu)=0.83 M_{1 / 2}, \\
\left(Y_{l}\right)_{i j}(\mu) & =1.5\left(Y_{l}\right)_{i j}\left(M_{X}\right), \\
\left(m_{L L}^{2}\right)_{i j}(\mu) & =\left(m_{L L}^{2}\right)_{i j}\left(M_{X}\right)+0.50\left|M_{1 / 2}\right|^{2} \delta_{i j}, \\
\left(m_{R R}^{2}\right)_{i j}(\mu) & =\left(m_{R R}^{2}\right)_{i j}\left(M_{X}\right)+0.15\left|M_{1 / 2}\right|^{2} \delta_{i j}, \\
\left(A_{l}\right)_{i j}(\mu) & =1.5\left(A_{l}\right)_{i j}\left(M_{X}\right)+0.67\left(Y_{l}\right)_{i j}(\mu) M_{1 / 2},
\end{aligned}
$$

where the low-energy values are evaluated at the renormalization point $\mu=500 \mathrm{GeV}$, and we neglect the effect of the Yukawa couplings, which is valid as far as the tau Yukawa couplings are small. For simplicity, we fix the $S_{3}$ breaking parameters at $\delta_{l}=6.577 \times 10^{-2}$ and $\epsilon_{l}=4.792 \times 10^{-3}$ with $y_{0}^{l}\langle H\rangle=2456 \mathrm{MeV}$ and $r_{R}=1$ (See eq. (48)). Contours of the branching ratio of $\mu \rightarrow e \gamma$ on the $m_{0}-M_{1 / 2}$ plane are depicted in Fig. 2. Here we consider the two cases. In Fig. 2 (a), we take $\rho_{R}=1, a_{1}^{R}=0$, and thus the $\left(m_{R R}^{2}\right)_{12}$ is the dominant source of lepton flavor violation. On the contrary, Fig. 2 (b), is the case where $\rho_{R}=0.1, a_{1}^{R}=m_{0}$ so that the $\left(m_{R L}^{2}\right)_{12}$ dominates over the other. We should compare the contours of the branching ratio with the expected sensitivity of the branching ratio $\sim 10^{-14}$, which is aimed by the MEG collaboration at PSI [26]. The both figures show that $\mu \rightarrow e \gamma$ will be detectable if the gaugino mass and the scalar mass are smaller than about $300 \mathrm{GeV}$. We note that the branching ratios are small when the universal scalar mass is small. This is understood easily if we notice that the lepton flavor violation is inherent in the scalar masses, and in this limit the effect is diluted by the large gaugino contributions coming from the renormalization group evolution. We also checked other choices of $\rho_{R}$ and $a_{1}$. Generically, the results are not sensitive to the these values. However, for a certain choice of the parameters, $a_{1}^{R} \sim \rho_{R} m_{0}$, an accidental cancellation between the two contributions to the lepton flavor violation can take place, and thus branching ratio will be significantly reduced.

In our analytic as well as numerical computations, we only considered the gaugino loops depicted as Fig. 1. This is valid for small $\tan \beta$. In fact there are other diagrams which generically enhance the branching ratio when $\tan \beta$ is large. Furthermore, it was pointed out in [27] that the contributions coming from various diagrams partially cancel with each other in certain regions of the parameter space. This cancellation is, however, very sensitive to the soft masses as well as the higgsino mass parameter, and it would be premature to make a full analysis with various unknown parameters in the model.

Bearing these observations in mind, we conclude that $\mu \rightarrow e \gamma$ is generically within the reach of the planning experiment in a wide range of the parameter space if the masses of the superparticles involved in the process are around the weak scale.

Here we would like to emphasize that considerable lepton flavor violation is expected in our scenario, even if the Yukawa couplings of the right-handed neutrinos are small and their 
effects in the RG running are negligible. This clearly contrasts with other cases considered previously $[23,24,28]$, where the $\mathrm{RG}$ effect from the right-handed neutrino Yukawa couplings is essential for sufficient lepton flavor violation.

Let us next consider other lepton flavor violating processes, $\tau \rightarrow \mu \gamma$ and $\tau \rightarrow e \gamma$. The ratios of the branching fractions are estimated as

$$
\frac{\operatorname{Br}(\tau \rightarrow \mu \gamma)}{\operatorname{Br}(\mu \rightarrow e \gamma)} \sim 0.17\left(\frac{\Delta_{l}}{\Delta_{l} E_{l}}\right)^{2} \sim 10^{3}, \quad \frac{\operatorname{Br}(\tau \rightarrow e \gamma)}{\operatorname{Br}(\mu \rightarrow e \gamma)} \sim 0.17\left(\frac{E_{l}}{\Delta_{l} E_{l}}\right)^{2} \sim 10
$$

where the factor 0.17 is the branching ratio of $\tau \rightarrow \mu \nu_{\tau} \bar{\nu}_{\mu}\left(e \nu_{\tau} \bar{\nu}_{e}\right)$. Recalling the fact that the current experimental bound of $\operatorname{Br}(\mu \rightarrow e \gamma)$ is already $\sim 10^{-11}$, we find that $\operatorname{Br}(\tau \rightarrow \mu \gamma)$ expected in our scenario can be at best $10^{-8}$, roughly two orders of magnitudes lower than its present experimental bound [29], and it may also be challenging for a Super B factory, which is to explore down to $10^{-7}-10^{-8}$ [30].

Finally, we would like to mention CP phases from the slepton sector. Complex phases in the slepton masses generally induce $\mathrm{CP}$ violation. The strongest constraint on the complex phases comes from the electron electric dipole moment (EDM) [31]:

$$
\left|\operatorname{Im}\left(\delta_{11}^{l}\right)_{L R}\right|<(\text { a few }) \times 10^{-8}\left(\frac{m_{\tilde{l}}}{100 \mathrm{GeV}}\right)^{2} .
$$

As is evident in eq. (47), $\left(m_{11}^{l 2}\right)_{L R}$ has two terms in our scenario: the first term is a universal one, and the second term is flavor-dependent term which originates from the democratic part of the Kähler potential. We find that the former contribution dominates, whose magnitude is estimated as

$$
\left(\delta_{11}^{l}\right)_{L R} \sim m_{e} a_{0} / m_{\tilde{l}}^{2} \sim 5 \times 10^{-6}
$$

To survive the experimental constraint, the phase should be rather small as $\sim 0.1-0.01$. We note that this universal contribution exists in many types of SUSY breaking scenario, including the minimal supergravity model, and the suppression of the phase of the universal $A$-term is a common problem in supersymmetry. ${ }^{4}$ Although our scenario does not solve this problem, it does not worsen the situation either.

\section{FCNC IN QUARK SECTOR}

Finally, we would like to briefly discuss FCNC constraints in the quark sector in a similar manner as in the previous section. Let us consider the $K, B$ and $D$ mass differences $\left(\Delta m_{K}, \Delta m_{B}\right.$ and $\left.\Delta m_{D}\right)$ and the branching fraction of the $b \rightarrow s \gamma$ process. They provide the upper bounds of off-diagonal elements of squark masses, which are listed in Table I. Here we quote the estimates in Ref. [19], which considers only gluino exchange diagrams, and all squark and gluino masses are fixed at $m_{\tilde{q}}=500 \mathrm{GeV}$ for simplicity. The limits are

\footnotetext{
${ }^{4}$ The CP phase of the Higgs mixing mass term should also be suppressed. See a recent proposal on this issue [32].
} 
scaled as $\left(m_{\tilde{q}} / 500 \mathrm{GeV}\right)$ for $\Delta m_{K}, \Delta m_{B}$ and $\Delta m_{D}$ and $\left(m_{\tilde{q}} / 500 \mathrm{GeV}\right)^{2}$ for $b \rightarrow s \gamma$. The magnitudes of the off-diagonal elements expected in this model are also listed in Table I. When compared with the experimental bounds, we conclude that FCNC processes induced by the $S_{3}$ symmetry breaking effect reside within the experimental bounds.

As for $b \rightarrow s \gamma$, there are other dangerous contributions from a chargino-stop loop and a charged Higgs loop. It is known that these two can contribute destructively for a sign of the higgsino mixing parameter $\mu$, and thus in certain regions of the parameter space the whole SUSY contribution is in accord with the experimental constraint. We expect that this occurs and will not discuss this point further.

The CP violating quantities $\epsilon_{K}, \epsilon^{\prime} / \epsilon$ of the kaon system put severer constraints on the imaginary parts of the flavor mixing masses. One finds that the constraint from the $\epsilon_{K}$ bound to the product $\left(\delta_{12}^{d}\right)_{L L}\left(\delta_{12}^{d}\right)_{R R}$ is much stronger than those to $\left(\delta_{12}^{d}\right)_{L L}$ or $\left(\delta_{12}^{d}\right)_{R R}$ :

$$
\sqrt{\left|\operatorname{Im}\left(\delta_{12}^{d}\right)_{L L}\left(\delta_{12}^{d}\right)_{R R}\right|}<2.2 \times 10^{-4}\left(\frac{m_{\tilde{q}}}{500 \mathrm{GeV}}\right) .
$$

On the other hand, the magnitude of this combination is generically expected to be

$$
\sqrt{\left(\delta_{12}^{d}\right)_{L L}\left(\delta_{12}^{d}\right)_{R R}} \sim \Delta_{d} E_{d} \sim 10^{-3}
$$

one order of magnitude above the experimental constraint, which may require some fine tuning to suppress the complex phase. Here, however, we argue that this constraint is easily overcome if the model is $\mathrm{SU}(5)$ inspired. In fact, if the model is embedded into $\mathrm{SU}(5)$, the right-handed down-type squark mass matrix is forced to become an almost unit matrix as well as the left-handed slepton one. This is simply because the two are in $5^{*}$ representation in the $\mathrm{SU}(5)$ and the left-handed sleptons are argued to have essentially no flavor violation, which is suggested by the bi-large neutrino mixing angles. Then $\left(\delta_{12}^{d}\right)_{R R}$ is zero, and hence the gluino contribution to $\epsilon_{K}$ vanishes. The upper bounds from the experiments and the expectations in our model for other processes are listed in Table II for the SU(5) case. From this table, one finds that our scenario marginally survives the bounds from the CP violating quantities.

\section{CONCLUSIONS AND DISCUSSION}

In this paper, we have proposed the extension of the democratic ansatz to the sector of superpartners of fermions (sfermions) in the Standard Model. It has been shown that the sfermion masses and their mixing controlled by the democratic ansatz solves the SUSY flavor problem and provides some phenomenologically interesting consequences.

More concretely we have postulated that the Kähler potential for the chiral supermultiplets of the quarks and leptons possesses the underlying $S_{3}$ symmetries of the democratic principle while the superpotential contains their small violation to obtain realistic Yukawa interactions for the quarks and leptons. Furthermore we have assumed that the hidden sector which is responsible to the SUSY breaking couples to the MSSM sector only in the Kähler potential, which is actually a usual assumption in the hidden sector SUSY breaking scenario.

In this framework, we have shown that the expectations from the $S_{3}$ symmetries and their violation are 1) the masses of the third generation sfermions can be different from 
those of the corresponding first two generation sfermions, and 2) the small violation of the $S_{3}$ symmetry induces the flavor violation in the sfermion sector. Remarkably the requirement that the neutrino sector should have bi-large mixing angles constrains the form of the Kähler metric of the left-handed lepton doublets. Unlike the general expectations given above, the requirement implies that the left-handed sleptons should have degenerate masses among the different generations, and also no flavor violation should arise in the left-handed slepton mass matrix.

This observation brings interesting phenomenological implications. The slepton mass spectrum should be of a special form. The left-handed sleptons have degenerate masses, while in the right-handed slepton sector the third generation will have a different mass from the first two generations. These masses are given at some high energy scale and suffer from radiative corrections. We expect that renormalization group analysis will relate the masses which will be measured experimentally with the masses given here. Thus we hope that the mass measurement of the slepton masses which is expected to be done in future collider experiments such as an $e^{+} e^{-}$linear collider will provide a test of the democratic sfermion ansatz.

A special attention has been paid to the lepton flavor violating process $\mu \rightarrow e \gamma$. We have shown that the branching ratio expected in our scenario survives the current experimental bound, and more interestingly it is generically within the reach of the forthcoming experiment [26] whose goal is to explore the branching ratio down to $\sim 10^{-14}$, if the sleptons and the charginos/neutralinos have masses around the weak scale. Furthermore, in our scenario, the lepton flavor violating operator of the dipole moment type appears only for the right-handed anti-muon, and thus this property can be tested if the polarized muon beam is available. On the other hand, the lepton flavor violation of the $\tau$ decay may not be a very promising process to see a signal of our scenario.

We should emphasize that the origin of the lepton flavor violation in the democratic sfermion ansatz is in the non-universality of the sfermion masses allowed by the democratic principle. This should be compared with the other mechanism to generate the lepton flavor violation that the renormalization group effects from the right-handed neutrino Yukawa interactions or the Yukawa interaction above the GUT scale induces the lepton flavor mixing in the slepton mass matrix which is assumed to be universal at the scale where these masses are originally generated.

\section{ACKNOWLEDGMENT}

This work was supported in part by the Grant-in-aid from the Ministry of Education, Culture, Sports, Science and Technology, Japan, No.12047201. KH thanks the Japan Society for the Promotion of Science for financial support.

\section{APPENDIX A: LFV DECAY RATES AND ELECTRON EDM}

In this appendix, we calculate lepton flavor violating decay rates and the EDM of the electron, which arise from dipole moment operators. 
We calculate the dipole moment operators using the mass insertion method. Here we discard higgsino-wino mixing diagrams ( $\mu$-term contribution) and double mass insertion diagrams (contributions from e.g., $\left.\left(m_{L L}^{2}\right)_{i j} \times\left(m_{L R}^{2}\right)_{j j}\right)$, which are proportional to $\mu \tan \beta$. Thus expressions below are valid in low $\tan \beta$ region. The resulting dipole moment operators are given by

$$
\mathcal{L}_{\text {eff }}=e \frac{m_{l_{j}}}{2} \bar{l}_{i} \sigma_{\mu \nu} F^{\mu \nu}\left(L_{i j} P_{L}+R_{i j} P_{R}\right) l_{j}
$$

where

$$
\begin{aligned}
L_{i j}= & \frac{\alpha}{2 \pi \cos ^{2} \theta_{W}} \frac{\left(m_{R R}^{2}\right)_{i j}}{m_{\tilde{l}_{R}}^{4}} M\left(x_{R}^{B}\right)-\frac{1}{2} \frac{\alpha}{2 \pi \cos ^{2} \theta_{W}}\left(\frac{M_{\widetilde{B}}}{m_{l j}}\right) \frac{\left(m_{R L}^{2}\right)_{i j}}{m_{\tilde{l}_{L}}^{2} m_{\tilde{l}_{R}}^{2}} \frac{T\left(x_{L}^{B}\right)-T\left(x_{R}^{B}\right)}{x_{L}^{B}-x_{R}^{B}}, \\
R_{i j}= & \frac{1}{4} \frac{\alpha}{2 \pi \sin ^{2} \theta_{W}} \frac{\left(m_{L L}^{2}\right)_{i j}}{m_{\tilde{l}_{L}}^{4}}\left[\tan ^{2} \theta_{W} M\left(x_{L}^{B}\right)+M\left(x_{L}^{W}\right)+S\left(x_{L}^{W}\right)\right] \\
& -\frac{1}{2} \frac{\alpha}{2 \pi \cos ^{2} \theta_{W}}\left(\frac{M_{\widetilde{B}}}{m_{l j}}\right) \frac{\left(m_{L R}^{2}\right)_{i j}}{m_{\tilde{l}_{L}}^{2} m_{\tilde{l}_{R}}^{2}} \frac{T\left(x_{L}^{B}\right)-T\left(x_{R}^{B}\right)}{x_{L}^{B}-x_{R}^{B}} .
\end{aligned}
$$

Here $\alpha$ is the fine-structure constant, $\sin \theta_{W}$ is the weak-mixing angle, $G_{F}$ is the Fermi constant. $m_{\tilde{l}_{L, R}}$ represent the average of slepton masses of left- and right-handed types. We define

$$
x_{L}^{B} \equiv \frac{M_{\widetilde{B}}^{2}}{m_{\tilde{l}_{L}}^{2}}, \quad x_{R}^{B} \equiv \frac{M_{\widetilde{B}}^{2}}{m_{\tilde{l}_{R}}^{2}}, \quad x_{L}^{W} \equiv \frac{M_{\widetilde{W}}^{2}}{m_{\tilde{l}_{L}}^{2}},
$$

and the functions of $x$ :

$$
\begin{aligned}
M(x) & \equiv \frac{1-9 x-9 x^{2}+17 x^{3}-6(3+x) x^{2} \log x}{12(1-x)^{5}} \\
S(x) & \equiv \frac{-1-9 x+9 x^{2}+x^{3}-6(1+x) x \log x}{3(1-x)^{5}} \\
T(x) & \equiv \frac{-x+x^{3}-2 x^{2} \log x}{2(x-1)^{3}}
\end{aligned}
$$

From the above expressions, we obtain the branching ratios for the processes $l_{j} \rightarrow l_{i} \gamma$, which are given by

$$
\operatorname{Br}\left(l_{j} \rightarrow l_{i} \gamma\right)=\frac{48 \pi^{3} \alpha}{G_{F}^{2}}\left(\left|L_{i j}\right|^{2}+\left|R_{i j}\right|^{2}\right) \times \operatorname{Br}\left(l_{j} \rightarrow l_{i} \bar{\nu}_{i} \nu_{j}\right),
$$

and the EDM of the electron by

$$
\frac{d_{e}}{e}=-m_{e} \operatorname{Im}\left(R_{11}\right)=\frac{\alpha M_{\widetilde{B}}}{4 \pi \cos ^{2} \theta_{W}} \frac{\operatorname{Im}\left[\left(m_{L R}^{2}\right)_{11}\right]}{m_{\tilde{l}_{L}}^{2} m_{\tilde{l}_{R}}^{2}} \frac{T\left(x_{L}^{B}\right)-T\left(x_{R}^{B}\right)}{x_{L}^{B}-x_{R}^{B}} .
$$




\section{REFERENCES}

[1] Y. Nir and N. Seiberg, Phys. Lett. B 309 (1993) 337 [arXiv:hep-ph/9304307];

M. Leurer, Y. Nir and N. Seiberg, Nucl. Phys. B 420 (1994) 468 [arXiv:hep-ph/9310320].

[2] M. Dine, R. G. Leigh and A. Kagan, Phys. Rev. D 48 (1993) 4269 [arXiv:hepph/9304299].

[3] P. Pouliot and N. Seiberg, Phys. Lett. B 318 (1993) 169 [arXiv:hep-ph/9308363].

[4] D. B. Kaplan and M. Schmaltz, Phys. Rev. D 49 (1994) 3741 [arXiv:hep-ph/9311281].

[5] A. Pomarol and D. Tommasini, Nucl. Phys. B 466 (1996) 3 [arXiv:hep-ph/9507462].

[6] R. Barbieri, G. R. Dvali and L. J. Hall, Phys. Lett. B 377 (1996) 76 [arXiv:hep$\mathrm{ph} / 9512388]$;

R. Barbieri, L. J. Hall, S. Raby and A. Romanino, Nucl. Phys. B 493 (1997) 3 [arXiv:hep-ph/9610449].

[7] L. J. Hall and H. Murayama, Phys. Rev. Lett. 75 (1995) 3985 [arXiv:hep-ph/9508296]; C. D. Carone, L. J. Hall and H. Murayama, Phys. Rev. D 53 (1996) 6282 [arXiv:hep$\mathrm{ph} / 9512399]$.

[8] H. Harari, H. Haut and J. Weyers, Phys. Lett. B 78 (1978) 459.

[9] Y. Koide, Phys. Rev. D 28 (1983) 252;

Y. Koide, Phys. Rev. D 39 (1989) 1391.

[10] H. Fritzsch and Z. Z. Xing, Phys. Lett. B 372 (1996) 265 [arXiv:hep-ph/9509389];

M. Fukugita, M. Tanimoto and T. Yanagida, Phys. Rev. D 57 (1998) 4429 [arXiv:hepph/9709388];

M. Fujii, K. Hamaguchi and T. Yanagida, Phys. Rev. D 65 (2002) 115012 [arXiv:hep$\mathrm{ph} / 0202210]$.

[11] T. Watari and T. Yanagida, Phys. Lett. B 544 (2002) 167 [arXiv:hep-ph/0205090].

[12] H. Fritzsch, Phys. Lett. B 73 (1978) 317.

[13] M. Kakizaki and M. Yamaguchi, in preparation.

[14] Y. Fukuda et al. [Super-Kamiokande Collaboration], Phys. Rev. Lett. 81 (1998) 1562 [arXiv:hep-ex/9807003].

[15] Y. Fukuda et al. [Super-Kamiokande Collaboration], Phys. Rev. Lett. 81 (1998) 1158 [Erratum-ibid. 81 (1998) 4279] [arXiv:hep-ex/9805021];

S. Fukuda et al. [Super-Kamiokande Collaboration], Phys. Rev. Lett. 86 (2001) 5656 [arXiv:hep-ex/0103033];

Q. R. Ahmad et al. [SNO Collaboration], Phys. Rev. Lett. 89 (2002) 011301 [arXiv:nuclex/0204008].

[16] M. Apollonio et al. [CHOOZ Collaboration], Phys. Lett. B 466 (1999) 415 [Erratumibid. B 472 (2000) 434] [arXiv:hep-ex/9907037].

[17] A. Suzuki, talk at XXI Symposium on Relativistic Astrophysics, Firenze, Italy (December 2002); K. Eguchi et al. [KamLAND collaboration], arXiv:hep-ex/0212021.

[18] L. J. Hall, J. Lykken and S. Weinberg, Phys. Rev. D 27 (1983) 2359.

[19] F. Gabbiani, E. Gabrielli, A. Masiero and L. Silvestrini, Nucl. Phys. B 477 (1996) 321 [arXiv:hep-ph/9604387].

[20] T. Moroi, Phys. Lett. B 321 (1994) 56.

[21] R. Barbieri and L. J. Hall, Phys. Lett. B 338 (1994) 212 [arXiv:hep-ph/9408406].

[22] M. L. Brooks et al. [MEGA Collaboration], Phys. Rev. Lett. 83 (1999) 1521 [arXiv:hepex/9905013]. 
[23] F. Borzumati and A. Masiero, Phys. Rev. Lett. 57 (1986) 961.

[24] J. Hisano, T. Moroi, K. Tobe, M. Yamaguchi and T. Yanagida, Phys. Lett. B 357 (1995) 579 [arXiv:hep-ph/9501407];

J. Hisano, T. Moroi, K. Tobe and M. Yamaguchi, Phys. Rev. D 53 (1996) 2442 [arXiv:hep-ph/9510309].

[25] Y. Kuno and Y. Okada, Phys. Rev. Lett. 77 (1996) 434 [arXiv:hep-ph/9604296].

[26] L. M. Barkov et al., Research Proposal for experiment at PSI.

[27] J. Hisano, T. Moroi, K. Tobe and M. Yamaguchi, Phys. Lett. B 391 (1997) 341 [Erratum-ibid. B 397 (1997) 357] [arXiv:hep-ph/9605296].

[28] J. Hisano, D. Nomura and T. Yanagida, Phys. Lett. B 437 (1998) 351 [arXiv:hep$\mathrm{ph} / 9711348]$

J. Hisano and D. Nomura, Phys. Rev. D 59 (1999) 116005 [arXiv:hep-ph/9810479].

[29] S. Ahmed et al. [CLEO Collaboration], Phys. Rev. D 61 (2000) 071101 [arXiv:hepex/9910060].

[30] T. Ohshima, talk at the workshop "Neutrino oscillations and their origin" (NOON2001) (ICRR, Univ. of Tokyo, Kashiwa, Japan, Dec.,2001).

[31] B. C. Regan, E. D. Commins, C. J. Schmidt and D. DeMille, Phys. Rev. Lett. 88 (2002) 071805.

[32] M. Yamaguchi and K. Yoshioka, Phys. Lett. B 543 (2002) 189 [arXiv:hep-ph/0204293]. 


\section{FIGURES}
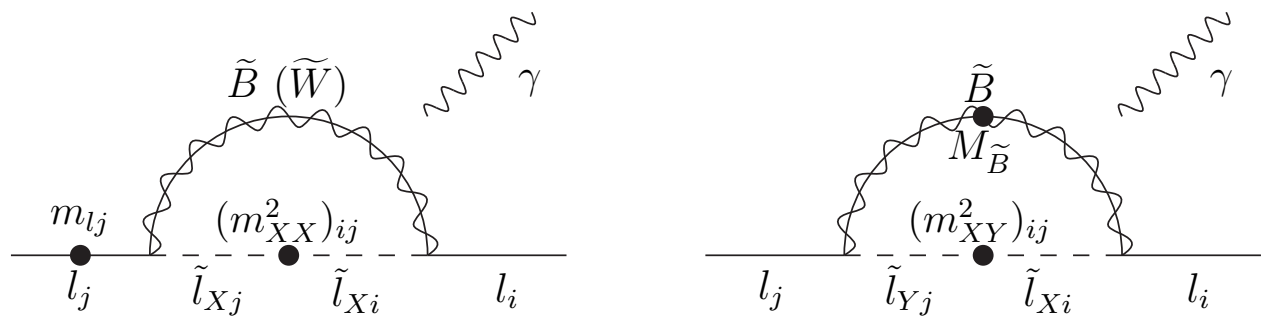

FIG. 1. Feynman diagrams which cause lepton flavor violating decays $l_{j} \rightarrow l_{i} \gamma . l_{i}, \tilde{l}_{X i}, \gamma, \widetilde{W}$ and $\widetilde{B}$ represent the charged leptons, charged sleptons, the photon, wino and bino, respectively. $m_{l j}$ and $M_{\widetilde{B}}$ are the masses of the charged leptons and bino. The blobs in the slepton lines indicate the insertions of the flavor violating mass matrix elements, $\left(m_{X X}^{2}\right)_{i j}$ and $\left(m_{X Y}^{2}\right)_{i j}$, and ones in the fermion lines indicate chirality flips of the charged leptons and bino. $X, Y$ stand for the chiralities $L$ or $R$.

(a) $\rho_{R}=1, a_{1}^{R}=0$

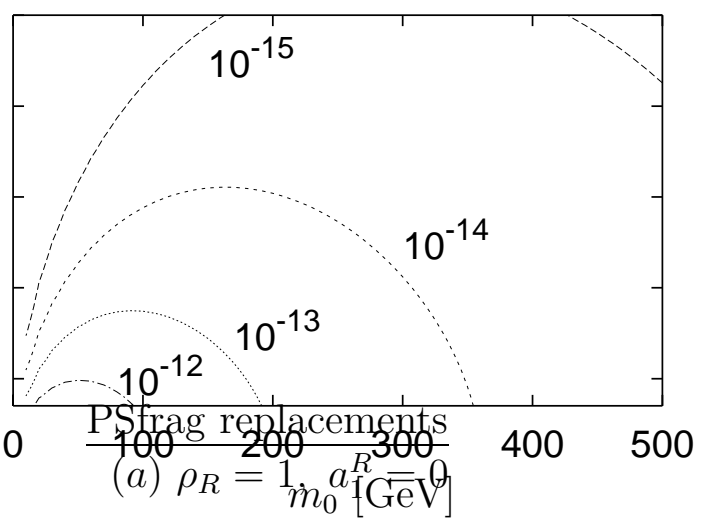

(b) $\rho_{R}=0.1, a_{1}^{R}=m_{0}$

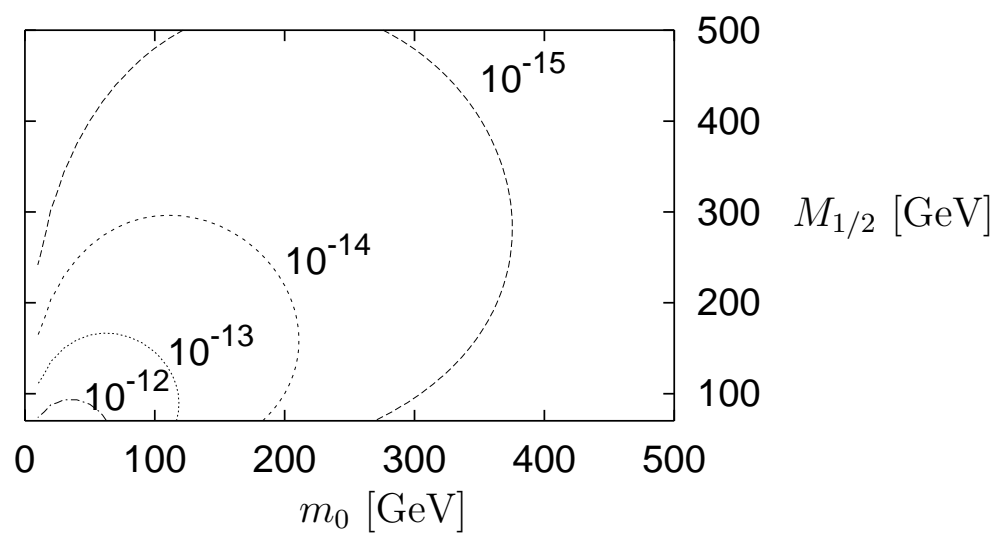

FIG. 2. Contour plots for $\operatorname{Br}(\mu \rightarrow e \gamma)$ in the $m_{0}-M_{1 / 2}$ plane for $(a) \rho_{R}=1, a_{1}^{R}=0$ and (b) $\rho_{R}=0.1, a_{1}^{R}=m_{0}$. The lines correspond to $\operatorname{Br}(\mu \rightarrow e \gamma)=10^{-12}, 10^{-13}, 10^{-14}$ and $10^{-15}$ from the lower left. Here, we fix the $S_{3}$ breaking parameters at $\delta_{l}=6.577 \times 10^{-2}$ and $\epsilon_{l}=4.792 \times 10^{-3}$ with $y_{0}^{l}\langle H\rangle=2456 \mathrm{MeV}$ and $r_{R}=1$. 


\section{TABLES}

TABLE I. Limits on $\delta$ 's obtained from the FCNC experiments and estimation in our model. We fix all squark masses $m_{\tilde{q}}$, gluino masses and $a_{1}$ 's at $500 \mathrm{GeV}$ as representative values. For other values of $m_{\tilde{q}}$, the limits can be obtained multiplying the ones in the table by $\left(m_{\tilde{q}} / 500 \mathrm{GeV}\right)$ for $\Delta m_{K}, \Delta m_{B}$ and $\Delta m_{D}$ and $\left(m_{\tilde{q}} / 500 \mathrm{GeV}\right)^{2}$ for $b \rightarrow s \gamma$. Notice that $\left(\delta_{i j}\right)_{L R} \sim\left(\delta_{i j}\right)_{R L}$ are imposed in obtaining the limits. It does not matter since we focus on order of magnitudes.

$$
\begin{aligned}
& \text { Exp. bounds [19] Our model } \\
& \Delta m_{K}
\end{aligned}
$$

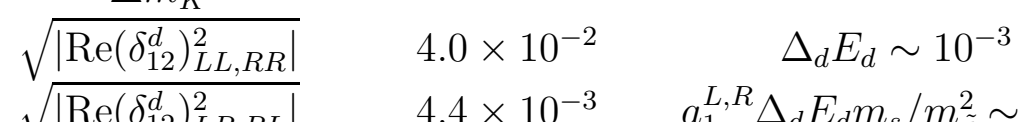

$$
\begin{aligned}
& \sqrt{\left|\operatorname{Re}\left(\delta_{12}^{d}\right)_{L R, R L}^{2}\right|} \quad 4.4 \times 10^{-3} \quad a_{1}^{L, R} \Delta_{d} E_{d} m_{s} / m_{\tilde{d}}^{2} \sim 10^{-6} \\
& \sqrt{\left|\operatorname{Re}\left(\delta_{12}^{d}\right)_{L L}\left(\delta_{12}^{d}\right)_{R R}\right|} \quad 2.8 \times 10^{-3} \quad \Delta_{d} E_{d} \sim 10^{-3} \\
& \begin{array}{ccc}
\Delta m_{B} & E_{d} \sim 10^{-2} \\
\sqrt{\left|\operatorname{Re}\left(\delta_{13}^{d}\right)_{L L, R R}^{2}\right|} & 9.8 \times 10^{-2} & a_{1}^{L, R} E_{d} m_{b} / m_{\tilde{d}}^{2} \sim 10^{-4} \\
\sqrt{\left|\operatorname{Re}\left(\delta_{13}^{d}\right)_{L R, R L}^{2}\right|} & 3.3 \times 10^{-2} & E_{d} \sim 10^{-2}
\end{array} \\
& \sqrt{\left|\operatorname{Re}\left(\delta_{12}^{u}\right)_{L L, R R}^{2}\right|} \\
& \sqrt{\left|\operatorname{Re}\left(\delta_{12}^{u}\right)_{L R, R L}^{2}\right|} \\
& \sqrt{\left|\operatorname{Re}\left(\delta_{12}^{u}\right)_{L L}\left(\delta_{12}^{u}\right)_{R R}\right|} \\
& \begin{array}{l}
3.1 \times 10^{-2} \\
1.7 \times 10^{-2}
\end{array} \\
& \Delta_{u} E_{u} \sim 10^{-6} \\
& a_{1}^{L, R} \Delta_{u} E_{u} m_{c} / m_{\tilde{u}}^{2} \sim 10^{-8} \\
& b \rightarrow s \gamma \\
& \left|\left(\delta_{23}^{d}\right)_{L L, R R}\right| \\
& \left|\left(\delta_{23}^{d}\right)_{L R, R L}\right| \\
& 1.6 \times 10^{-2} \\
& \Delta_{u} E_{u} \sim 10^{-6} \\
& \Delta_{d} \sim 10^{-1} \\
& a_{1}^{L, R} \Delta_{d} m_{b} / m_{\tilde{u}}^{2} \sim 10^{-3}
\end{aligned}
$$


TABLE II. Limits on $\delta$ 's obtained from the $\mathrm{CP}$ violating parameters and estimation in our model in the SU(5) GUT inspired case. We use the same parameters as in Table I. For other values of $m_{\tilde{q}}$, the limits can be obtained multiplying the ones in the table by $\left(m_{\tilde{q}} / 500 \mathrm{GeV}\right)$ for $\epsilon_{K}$ and $\left(m_{\tilde{q}} / 500 \mathrm{GeV}\right)^{2}$ for $\epsilon^{\prime} / \epsilon$.

$$
\begin{array}{ccc} 
& \text { Exp. bounds [19] } & \text { Our model } \\
\epsilon_{K} & 3.2 \times 10^{-3} & \Delta_{d} E_{d} \sim 10^{-3} \\
\sqrt{\left|\operatorname{Im}\left(\delta_{12}^{d}\right)_{L L}^{2}\right|} & 3.2 \times 10^{-3} & \sim 0 \\
\sqrt{\left|\operatorname{Im}\left(\delta_{12}^{d}\right)_{R R}^{2}\right|} & 3.5 \times 10^{-4} & a_{1}^{L} \Delta_{d} E_{d} m_{s} / m_{\tilde{d}}^{2} \sim 10^{-6} \\
\sqrt{\left|\operatorname{Im}\left(\delta_{12}^{d}\right)_{L R}^{2}\right|} & 3.5 \times 10^{-4} & a_{1}^{L} \Delta_{d} E_{d} m_{d} / m_{\tilde{d}}^{2} \sim 10^{-8} \\
\sqrt{\left|\operatorname{Im}\left(\delta_{12}^{d}\right)_{R L}^{2}\right|} & 2.2 \times 10^{-4} & \sim 0 \\
\sqrt{\left|\operatorname{Im}\left(\delta_{12}^{d}\right)_{L L}\left(\delta_{12}^{d}\right)_{R R}\right|} & & \\
\epsilon^{\prime} / \epsilon & 0.48 & \Delta_{l} E_{l} \sim 10^{-3} \\
\left|\operatorname{Im}\left(\delta_{12}^{d}\right)_{L L}\right| & 0.48 & \sim 0 \\
\left|\operatorname{Im}\left(\delta_{12}^{d}\right)_{R R}\right| & 2.0 \times 10^{-5} & a_{1}^{L} \Delta_{d} E_{d} m_{s} / m_{\tilde{l}}^{2} \sim 10^{-6} \\
\left|\operatorname{Im}\left(\delta_{12}^{d}\right)_{L R}\right| & 2.0 \times 10^{-5} & a_{1}^{L} \Delta_{l} E_{l} m_{d} / m_{\tilde{l}}^{2} \sim 10^{-8} \\
\left|\operatorname{Im}\left(\delta_{12}^{d}\right)_{R L}\right| & &
\end{array}
$$

